\title{
Switching statin-treated patients from fenofibrate to the prescription omega-3 therapy icosapent ethyl: a retrospective case series
}

\author{
Richard S. Castaldo ${ }^{1,2}$
}

Published online: 8 March 2016

(C) The Author(s) 2016. This article is published with open access at Springerlink.com

\begin{abstract}
Introduction Patients receiving statin therapy for dyslipidaemia often require treatment with an additional agent to control triglyceride levels. Options for add-on therapy include fibrates and omega-3 fatty acids. This case series describes the effects of switching add-on therapy from fenofibrate to icosapent ethyl (the ethyl ester of the omega3 fatty acid, eicosapentaenoic acid) on patient lipid profiles. Methods This was a retrospective analysis of patient records from a private medical practice in western New York. Statin-treated patients with dyslipidaemia who had been treated with fenofibrate and later switched to icosapent ethyl were selected for analysis. Lipid profiles before and after the switch to icosapent ethyl were compared.

Results The records of five patients were analysed. All patients had hypertension and were overweight, male, and at high cardiovascular risk. After the switch to icosapent ethyl (treatment duration 3.9-5.8 months), triglyceride levels decreased in four patients, and low-density lipoprotein cholesterol, non-high-density lipoprotein cholesterol, and total cholesterol levels decreased in all patients. High-density lipoprotein levels increased in four patients. Icosapent ethyl was well tolerated.

Conclusions Switching from fenofibrate to icosapent ethyl as add-on to a statin therapy due to clinical need may provide an option for patients to maintain or improve lipid parameters.
\end{abstract}

Richard S. Castaldo

jeanettectitus@yahoo.com

1 Niagara Falls Memorial Medical Center, North Tonawanda, NY, USA

2 Internal Medicine, PO Box 37, Arcade, NY 14009, USA

\section{Introduction}

Treatment of dyslipidaemia with statins is effective for lowering low-density lipoprotein cholesterol (LDL-C) levels and can improve levels of other lipids [1, 2]. However, triglyceride (TG) levels in statin-treated patients may remain uncontrolled, leaving patients at residual risk for cardiovascular events [3]. Fibrates, niacin, and prescription omega3 fatty acids are indicated to reduce TG levels in patients with severe $(\geq 500 \mathrm{mg} / \mathrm{dL})$ hypertriglyceridaemia, and may be options as add-on therapy to statins in patients with persistently elevated TG levels [4]. Fibrates, available since the 1990s, effectively lower TG levels, but safety issues, such as the potential for myopathy and unwanted effects on LDL-C, may warrant reassessment of therapeutic use, particularly in some patients receiving statin therapy [5-8].

In 2012, icosapent ethyl (Vascepa ${ }^{\circledR}$; Amarin Pharma Inc., Bedminster, NJ), a high-purity prescription formulation containing the ethyl ester of eicosapentaenoic acid (EPA), was approved by the US FDA. Icosapent ethyl is indicated as an adjunct to diet to reduce TG levels in adult patients with severe $(\geq 500 \mathrm{mg} / \mathrm{dL}$ ) hypertriglyceridaemia [9]. EPA is the sole active ingredient in icosapent ethyl [9] and has been shown to reduce TG, non-high-density lipoprotein cholesterol (non-HDL-C), and total cholesterol (TC) levels without raising LDL-C levels in patients with very high TG levels ( $\geq 500$ and $\leq 2000 \mathrm{mg} / \mathrm{dL}$ ), as well as in statin-treated patients with residually high TG levels ( $\geq 200$ and $<500 \mathrm{mg} / \mathrm{dL}$ ) [10, 11]. Icosapent ethyl has been found to be safe and generally well tolerated, with a safety profile similar to that of placebo [9-11]. In doubleblind studies, arthralgia was the only adverse reaction reported to occur in $>2 \%$ of patients treated with icosapent ethyl and at an incidence rate greater than that for placebo [9]. 
Because of the robust safety and efficacy profile of icosapent ethyl, patients receiving fibrate therapy were gradually switched to treatment with icosapent ethyl in a private medical practice in western New York, a geographical area known for its high risk of heart disease and stroke [12]. The objective of this analysis was to retrospectively assess the lipid profile effects of switching statin-treated patients from fibrate therapy to icosapent ethyl.

\section{Methods}

\section{Study design}

This retrospective chart review was carried out at a single private medical practice with offices located in Franklinville, Tonawanda, Lancaster, and Niagara Falls, NY. Patient records were eligible for review if the patients had TG levels $\geq 500 \mathrm{mg} / \mathrm{dL}$ at some point in their medical history and if they were receiving stable fibrate therapy in addition to stable statin therapy and were switched from fibrate therapy to icosapent ethyl while continuing with stable statin therapy. Patients were required to have been treated with icosapent ethyl plus a statin for $>2$ months, have available lipid measurements, and have been clinically stable for the period encompassing when the lipid measurements were made while on fibrate therapy and while on icosapent ethyl. Patients were excluded if they had any gaps in treatment, missing lipid measurements, known non-compliance, or any changes in clinical condition or medications that could affect lipid parameters. Written informed consent was received from all patients included in this case series.

\section{Assessments}

Lipid parameters were assessed a minimum of 2 months after the initiation of fenofibrate therapy and a minimum of 2 months after the switch to icosapent ethyl. Blood samples for lipid analysis were collected from patients after an overnight fast and analysed by local laboratories as specified by patient insurance. Parameters measured included TG, LDL-C, HDL-C, TC, and non-HDL-C levels.

Body mass index was calculated as weight in kilograms divided by height in metres squared. LDL-C levels were calculated using the Friedewald formula [i.e. LDL-C $=$ TC minus HDL-C minus (TG/5)] in some instances (note, the Friedewald formula should not be used when TG levels are $>400 \mathrm{mg} / \mathrm{dL}$ ) [13]. Non-HDL-C levels were calculated as TC minus HDL-C [14]. Percentage changes in individual patient lipid parameters from before and after the switch from add-on fibrate therapy to treatment with icosapent ethyl were calculated.

\section{Results}

\section{Patients and treatments}

The medical records of five patients (aged 58-66 years) met the inclusion criteria and were analysed (Table 1). All patients were overweight men with hypertension and high cardiovascular risk. One patient had coronary heart disease and chronic kidney disease.

All patients were on stable doses of moderate-intensity statins, including lovastatin, extended-release fluvastatin, or atorvastatin therapy and subsequently initiated fibrate therapy between August 2007 and June 2010 (before the approval of icosapent ethyl). Fibrate therapy consisted of fenofibrate tablets $145 \mathrm{mg}$ once daily $\left(\right.$ Tricor $^{\circledR}$; AbbVie Inc., North Chicago, IL) in two patients, and fenofibrate capsules $150 \mathrm{mg}$ once daily (Lipofen ${ }^{\circledR}$; Kowa Pharmaceuticals America, Inc., Montgomery, AL) in the remaining three patients.

Add-on therapy was switched from fibrate therapy to icosapent ethyl between March and September 2014. After the switch, all patients received two capsules of icosapent ethyl twice daily, for a total dosage of $4 \mathrm{~g} /$ day in addition to their stable statin regimen. All patients had been treated with icosapent ethyl + statin therapy for $\geq 3.9$ months (range 3.9-5.8 months) before lipid measurements. Because of comorbidities, patients were also receiving other medications (antihypertensive agents: amlodipine/ olmesartan, atenolol, nebivolol, olmesartan, quinapril, verapamil; others: albuterol, aspirin, azelastine, cetirizine, levothyroxine, mometasone furoate, montelukast sodium, omeprazole).

\section{Lipid parameters}

TG levels ranged from 91 to $278 \mathrm{mg} / \mathrm{dL}$ during the period when patients were receiving fenofibrate + statin and from 81 to $190 \mathrm{mg} / \mathrm{dL}$ when they were receiving icosapent ethyl + statin (Table 1). Four patients experienced a decrease in TG level after switching from fenofibrate to icosapent ethyl, with decreases ranging from 8 to $53 \%$ (Table 1). The remaining patient had an increase of $4 \%$ in TG level, from $182 \mathrm{mg} / \mathrm{dL}$ while on fenofibrate + statin to $190 \mathrm{mg} / \mathrm{dL}$ while on icosapent ethyl + statin (Table 1).

While patients were receiving fenofibrate + statin, their LDL-C levels ranged from 55 to $170 \mathrm{mg} / \mathrm{dL}$ (Table 1). After the switch to icosapent ethyl, LDL-C levels decreased in all patients, with a maximum decrease of $44 \%$ in one patient (Table 1).

Reductions in non-HDL-C levels, ranging from 3 to $39 \%$, were observed in all patients following the switch from fenofibrate + statin to icosapent ethyl + statin 
Table 1 Characteristics and lipid levels of statin-treated patients switched from fibrate therapy to icosapent ethyl

\begin{tabular}{|c|c|c|c|c|c|}
\hline Parameter & Patient 1 & Patient 2 & Patient 3 & Patient 4 & Patient 5 \\
\hline \multicolumn{6}{|l|}{ Patient characteristics } \\
\hline Age (years) ${ }^{\mathrm{a}}$ & 58 & 63 & 61 & 64 & 66 \\
\hline Body mass index $\left(\mathrm{kg} / \mathrm{m}^{2}\right)$ & 26 & 28 & 30 & 33 & 28 \\
\hline Gender & Male & Male & Male & Male & Male \\
\hline Relevant conditions & $\begin{array}{l}\text { Hypertension; } \\
\text { hypothyroidism; } \\
\text { BPH }\end{array}$ & $\begin{array}{l}\text { Hypertension; } \\
\text { CHD; } \\
\text { CKD }\end{array}$ & $\begin{array}{l}\text { Hypertension; } \\
\text { GORD; } \\
\text { chronic } \\
\text { sinusitis }\end{array}$ & $\begin{array}{l}\text { Hypertension; } \\
\text { allergic } \\
\text { sinusitis; } \\
\text { asthma }\end{array}$ & $\begin{array}{l}\text { Hypertension; } \\
\text { arthralgia; } \\
\text { arthritis }\end{array}$ \\
\hline
\end{tabular}

\section{Treatment dosage and duration}

$\begin{array}{llllll}\text { Statin daily dosage }(\mathrm{mg}) & \text { Lovastatin } 40 & \text { Lovastatin } 40 & \begin{array}{c}\text { Fluvastatin ER } \\ 80\end{array} & \begin{array}{c}\text { Atorvastatin } 10 \\ \text { Atorvastatin } \\ 10\end{array} \\ \text { Fenofibrate daily dosage }(\mathrm{mg}) & 145 & 145 & 150 & 150 & 150 \\ \text { Time on fibrate }+ \text { statin (months) } & 17.7 & 17.9 & 29.4 & 29.0 & 4.7 \\ \text { Time on icosapent ethyl + statin } & 5.0 & 5.8 & 4.8 & 3.9 & 3.9\end{array}$
(months) ${ }^{c}$

Triglyceride levels $(\mathrm{mg} / \mathrm{dL})$

\begin{tabular}{llllll} 
Before statin & 545 & 564 & 509 & 522 & 592 \\
Statin alone & 245 & 279 & 271 & 300 & 217 \\
\hline Fibrate + statin & 278 & 167 & 182 & 123 & 91 \\
Icosapent ethyl + statin $^{\text {d change }}$ & 130 & 81 & 190 & 107 & 84 \\
\hline d $^{\text {d }}$ & -53.2 & -51.5 & +4.4 & -13.0 & -7.7
\end{tabular}

Low-density lipoprotein cholesterol levels $(\mathrm{mg} / \mathrm{dL})^{\mathrm{e}}$

\begin{tabular}{|c|c|c|c|c|c|}
\hline Before statin & 104 & 188 & NR & NR & 189 \\
\hline Statin alone & 96 & 126 & 84 & 180 & $88^{\mathrm{f}}$ \\
\hline Fibrate + statin & 55 & 170 & 121 & 151 & 115 \\
\hline Icosapent ethyl + statin & 54 & $96^{\mathrm{f}}$ & $105^{\mathrm{f}}$ & $135^{\mathrm{f}}$ & 96 \\
\hline$\%$ change $^{\mathrm{d}}$ & -1.8 & -43.5 & -13.2 & -10.6 & -16.5 \\
\hline \multicolumn{6}{|c|}{ Non-HDL-C levels $(\mathrm{mg} / \mathrm{dL})^{\mathrm{f}}$} \\
\hline Before statin & 202 & 189 & 212 & 228 & NR \\
\hline Statin alone & 153 & 137 & 138 & 200 & 131 \\
\hline Fibrate + statin & 105 & 184 & 148 & 175 & 131 \\
\hline Icosapent ethyl + statin & 77 & 112 & 143 & 155 & 127 \\
\hline$\%$ change $^{\mathrm{d}}$ & -26.7 & -39.1 & -3.4 & -11.4 & -3.1 \\
\hline \multicolumn{6}{|c|}{ Total cholesterol levels $(\mathrm{mg} / \mathrm{dL})$} \\
\hline Before statin & 232 & 235 & 235 & NR & 272 \\
\hline Statin alone & 188 & 185 & 168 & 243 & 184 \\
\hline Fibrate + statin & 136 & 231 & 177 & 211 & 178 \\
\hline Icosapent ethyl + statin & 112 & 160 & 172 & 201 & 175 \\
\hline$\%$ change $^{\mathrm{d}}$ & -17.7 & -30.7 & -2.8 & -4.7 & -1.7 \\
\hline \multicolumn{6}{|l|}{ HDL-C levels (mg/dL) } \\
\hline Before statin & 30 & 46 & 23 & 41 & 52 \\
\hline Statin alone & 35 & 48 & 30 & 43 & 53 \\
\hline Fibrate + statin & 31 & 47 & 29 & 36 & 47 \\
\hline
\end{tabular}


Table 1 continued

$\begin{array}{llllll}\text { Parameter } & \text { Patient } 1 & \text { Patient } 2 & \text { Patient 3 } & \text { Patient } 4 & \text { Patient } 5 \\ \text { Icosapent ethyl + statin }^{\text {d }} & 35 & 48 & 29 & 46 & 48 \\ \text { change }^{\mathrm{d}} & +12.9 & +2.1 & 0.0 & +27.8 & +2.1\end{array}$

$B P H$ benign prostatic hypertrophy, $C H D$ coronary heart disease, $C K D$ chronic kidney disease, $E R$ extended release, GORD gastro-oesophageal reflux disease, $H D L-C$ high-density lipoprotein cholesterol, $N R$ not reported

${ }^{a}$ Age at time of measurement while on icosapent ethyl

b Time from addition of fenofibrate to statin to when laboratory measurements were taken after initiation of fenofibrate

c Time from switch from fenofibrate + statin to icosapent ethyl + statin to when laboratory measurements were taken after icosapent ethyl was initiated

${ }^{d}$ Percentage changes in lipid levels from when patient was on fibrate therapy to when on icosapent ethyl

e Values were measured directly unless otherwise noted

f Value is calculated based on other information available (see "Methods" section)

(Table 1). TC levels decreased in all patients (range 2-31\%) following the switch from fenofibrate + statin to icosapent ethyl + statin (Table 1). An increase or no change in HDL-C level was observed in all patients (range $0-28 \%)$.

\section{Safety}

The switch from fenofibrate + statin to icosapent ethyl + statin was well tolerated by all patients, with no adverse effects reported. All patients are currently continuing on this regimen. No changes in arthritis or arthralgia were observed in one patient who had these conditions, which were pre-existing to omega-3 fatty acid therapy.

\section{Discussion}

In this real-world retrospective analysis of high-risk patients receiving statin therapy, patient medical records were examined to evaluate the potential lipid effects of switching from add-on fibrate therapy to the prescription omega-3 fatty acid therapy icosapent ethyl while continuing stable statin therapy. After switching from fenofibrate to icosapent ethyl, lipid control was maintained or improved, with all patients experiencing improvements in atherogenic lipid parameters, including reductions in TC, LDL-C, and non-HDL-C levels, and all but one patient experienced a reduction in TG levels. The slight $4 \%$ increase in TG levels in one patient (from 182 to $190 \mathrm{mg}$ / dL) was not considered to be clinically significant; it is not known what factors could have resulted in this increase, but this may have been due to variability in laboratory measurements or changes in patient diet, exercise, or medication adherence that were not reported during the office visit.
Historically, fibrate and niacin products have been used as adjunctive therapy to statins, with fibrates being more commonly prescribed for high TG levels. This is reflected in this case series, wherein TG levels improved in four of the five patients when fenofibrate was added to statin treatment. Nonetheless, potential safety concerns have been recognized with the use of fibrates or niacin in combination with statins. Several statin labels mention that use in combination with fibrates or lipid-lowering doses of niacin may increase the risk of adverse skeletal muscle effects; however, in large outcomes trials, these events were not very common with fibrates [15-19]. Some statin labels specifically recommend avoiding concomitant therapy with gemfibrozil and/or note an increased risk of adverse skeletal muscle effects with gemfibrozil [16, 17, 20]. Fibrates also may increase levels of serum transaminases and serum creatinine, the former in combination with statins [5, 6]. Importantly, fibrates may be associated with increases in LDL-C levels, especially in patients with very high TG levels, or attenuation of statin-induced reductions in LDL-C levels [1, 7, 8, 21-27]. While the exact mechanism of this increase in LDL-C levels is unknown, it may in part be due to an increase in plasma PCSK9 levels resulting from fibrate therapy [28-30]. With niacins, flushing is a common tolerability concern, and other potential adverse effects may include increased serum glucose and hepatotoxicity or myopathy, especially when co-administered with a statin [31].

A meta-analysis of 19 randomized studies found that fibrate use was not associated with a reduced risk of allcause or coronary heart disease mortality or stroke; these findings held true for the subset of studies in which fibrates were used in combination with statins, and, in this subset, fibrate therapy also did not reduce the risk of non-fatal myocardial infarction [32]. Supporting this, a retrospective analysis of patients with type 2 diabetes who were 
receiving statins alone or in combination with fibrate therapy showed that the use of combination therapy did not reduce the incidence of cardiovascular disease compared with the use of statins alone [33]. Notably, in April 2015, the US FDA removed the indication for use of fenofibric acid delayed-release capsules (Trilipix ${ }^{\circledR} ;$ AbbVie Inc.) and niacin extended-release tablets (Niaspan ${ }^{\circledR}$; AbbVie Inc.) in combination with statins from the product labels $[6,31$, 34]. Thus, other options are needed for add-on therapy with statins. The product label's 'limitations of use' notes that fenofibrate was not shown to reduce coronary heart disease morbidity and mortality in patients with type 2 diabetes. However, hypothesis-generating data from large cardiovascular outcomes trials suggest that patients with diabetes and atherogenic dyslipidaemia characterized by high TG and low HDL-C levels may benefit from treatment with fenofibrate $[18,19]$. This is yet to be proven in a large, prospective, randomized, placebo-controlled trial.

Prescription omega-3 fatty acid products first became commercially available in the USA in 2005 (Lovaza $^{\circledR}$; GlaxoSmithKline, Research Triangle Park, NC [35]) and contained a combination of both EPA and docosahexaenoic acid (DHA); additional EPA + DHA products were recently approved in 2014 (Omtryg ${ }^{\circledR}$; Trygg Pharma Inc., Arlington, VA [36], and Epanova ${ }^{\circledR}$; AstraZeneca Pharmaceuticals, Wilmington, DE [37]). Although these agents are also effective in lowering TG levels, their prescribing information warns that they may raise LDL-C levels [3537]. In pivotal clinical studies of patients with TG levels $\geq 500 \mathrm{mg} / \mathrm{dL}$, products that contain both EPA and DHA were found to increase LDL-C levels by $\approx 15$ to $49 \%$ versus placebo [35-37]. Meta-analyses and systematic reviews have found that EPA and DHA have differential effects on LDL-C, wherein DHA may significantly increase LDL-C levels while EPA may significantly decrease or have a neutral effect on LDL-C levels [38, 39]. Thus, omega- 3 fatty acid products containing DHA may not be an optimal choice for patients with dyslipidaemia, as they may have unwanted effects on LDL-C. As noted earlier, icosapent ethyl, a high-purity form of EPA, does not raise LDL-C levels in patients with very high TG levels $[9,10]$. Furthermore, in statin-treated patients with high TG $(\geq 200$ and $<500 \mathrm{mg} / \mathrm{dL})$ and well-controlled LDL-C $(<100 \mathrm{mg} / \mathrm{dL})$ levels, icosapent ethyl $4 \mathrm{~g} /$ day was found to significantly reduce LDL-C levels by $6 \%$ versus placebo $(p=0.007)$ [11]. In the current analysis of statin-treated patients, LDL-C level decreases of 2-44\% were observed in all patients following the switch from fenofibrate to icosapent ethyl. In other retrospective chart analyses and case reports, switching from prescription omega-3 fatty acid products containing EPA + DHA to icosapent ethyl also resulted in decreases in TG and LDL-C levels, as was observed in this analysis of patients switched from fenofibrate therapy to treatment with icosapent ethyl [4045].

In addition to TG-lowering effects, omega-3 fatty acids have other reported beneficial cardiovascular effects on inflammation, blood pressure, thrombosis, and arrhythmia [46]. Furthermore, EPA alone or in combination with a statin may exert pleiotropic beneficial effects in multiple steps of atherosclerosis, including antioxidant effects [47, 48]; endothelial function [49, 50]; effects on macrophages, monocytes, and foam cells [51-53]; inflammation [54, 55]; plaque progression, formation, and vulnerability [52, 53, 56-59]; and thrombus formation [60, 61]. Given that omega-3 fatty acid products containing DHA may raise LDL-C levels and are not considered to be therapeutically equivalent to icosapent ethyl, per FDA therapeutic equivalence codes, they should not be substituted for prescription icosapent ethyl [62]. It is worth noting that while many non-prescription omega-3 fatty acid products are available in the USA, these are dietary supplements, not over-thecounter medications. Dietary supplements are not required to prove or demonstrate safety or efficacy before marketing [63-65], and have been found to have highly variable product quality, purity, and EPA and DHA content within and between brands [66-69]. Dietary supplements may require a high pill burden to achieve prescription-strength therapeutic doses [70, 71], and they also may contain cholesterol, saturated fats [72, 73], contaminants such as polychlorinated biphenyl [69], and oxidation products [67, 74]. Given these considerations, it may be important to advise patients not to substitute omega-3 fatty acid dietary supplements for prescription icosapent ethyl.

While no adverse effects occurred in the patients in this assessment, safety and tolerability concerns regarding fibrate and niacin products, as noted earlier, have informed the switching of patients to safer, more tolerable options in my practice. In three patients, LDL-C levels were higher with fenofibrate + statin (170, 121, and $115 \mathrm{mg} / \mathrm{dL}$, respectively) than on a statin alone $(126,84$, and $88 \mathrm{mg} /$ $\mathrm{dL}$ ); an attenuation of LDL-C control after addition of fenofibrate to moderate- or high-intensity statins in patients with dyslipidaemia has been described in the literature $[8$, 21 , 27]. After the switch from fenofibrate + statin to icosapent ethyl + statin, LDL-C levels decreased to 96, 105 , and $96 \mathrm{mg} / \mathrm{dL}$, respectively. As noted, omega-3 fatty acid products containing DHA may potentially raise LDL$\mathrm{C}$ levels and have been associated with gastrointestinal concerns, such as eructation, dyspepsia, taste perversion, diarrhoea, nausea, abdominal pain or discomfort, and constipation, which may cause tolerability issues [35-37]. Thus, icosapent ethyl represents a TG-lowering option with fewer safety, tolerability, and efficacy concerns than other available choices. When combining an additional lipidlowering agent with a statin, icosapent ethyl may offer 
greater flexibility in adjusting or increasing statin doses than fenofibrate, given the potential safety issues and effects on LDL-C of fibrate agents.

As to whether addition of icosapent ethyl to statin therapy reduces cardiovascular risk, in the Japan EPA Lipid Intervention Study (JELIS) of 18,645 patients with hypercholesterolaemia, the ethyl ester of EPA, when added to statin therapy, significantly reduced the risk of a major coronary event by $19 \%$ compared with statin alone (hazard ratio $0.81 ; 95 \%$ confidence interval 0.69-0.95; $p=0.011$ ) [75]. While the dosage of EPA in the JELIS study was $1.8 \mathrm{~g} /$ day, the plasma levels achieved in JELIS $(169 \mu \mathrm{g} / \mathrm{mL})$ were similar to those achieved with icosapent ethyl $4 \mathrm{~g} /$ day $(183 \mu \mathrm{g} / \mathrm{mL})$ in statin-treated patients with TG levels $\geq 200$ and $<500 \mathrm{mg} / \mathrm{dL}$ [75, 76]. The ongoing, phase III, placebo-controlled Reduction of Cardiovascular Events with EPA-Intervention Trial (REDUCE-IT; ClinicalTrials.gov identifier NCT01492361) is investigating whether icosapent ethyl $4 \mathrm{~g} / \mathrm{day}$ in combination with statin therapy is more effective than statin alone in improving long-term cardiovascular outcomes in high-risk patients with hypertriglyceridaemia [77].

Based on clinical decisions or due to the recent removal of the indication for fenofibric acid use in combination with statin therapy from the product label (and the expectation of potentially similar revisions to the labels of generic products), clinicians may wish to consider a different statin add-on therapy for control of TGs and other lipids. The present exploratory analysis may be of interest for those wishing to identify other options for such patients.

The limitations of this case series include its small sample size, retrospective design, and patient heterogeneity regarding concomitant medications and underlying medical conditions. In real-world settings such as this, other limitations include lack of verification of patient compliance with fasting before lipid assessments, adherence to concomitant medications, and possible effects of changes in diet and exercise that were not reported to the physician. Larger, prospective studies are needed to confirm these hypothesis-generating findings.

\section{Conclusion}

Switching statin add-on therapy from fenofibrate to icosapent ethyl maintained or improved the lipid profile and was well tolerated with no adverse reactions in a series of patients with hypertension and high cardiovascular risk. Important differences between icosapent ethyl and other add-on therapy options include its good safety and tolerability profile and the fact that it does not increase LDL-C levels, as supported by clinical studies and the icosapent ethyl product label.
Acknowledgments Medical writing assistance was provided by Peloton Advantage, Parsippany, NJ, and funded by Amarin Pharma Inc., Bedminster, NJ. Medical scientific reference checks and associated assistance were provided by Sephy Philip, RPh, PharmD, and Joy Bronson of Amarin Pharma Inc.

\section{Compliance with ethical standards}

Funding R.S. Castaldo received reimbursement for the costs associated with data extraction from Amarin Pharma Inc.

Conflict of interest R.S. Castaldo declares having received payments for consulting and lecturing from Amarin Pharma Inc.

Open Access This article is distributed under the terms of the Creative Commons Attribution-NonCommercial 4.0 International License (http://creativecommons.org/licenses/by-nc/4.0/), which permits any noncommercial use, distribution, and reproduction in any medium, provided you give appropriate credit to the original author(s) and the source, provide a link to the Creative Commons license, and indicate if changes were made.

\section{References}

1. Jacobson TA, Ito MK, Maki KC, et al. National Lipid Association recommendations for patient-centered management of dyslipidemia: part 1-executive summary. J Clin Lipidol. 2014;8(5): 473-88.

2. Stone NJ, Robinson J, Lichtenstein AH, et al. 2013 ACC/AHA guideline on the treatment of blood cholesterol to reduce atherosclerotic cardiovascular risk in adults: a report of the American College of Cardiology/American Heart Association Task Force on Practice Guidelines. J Am Coll Cardiol. 2014;63(25 Pt B):2889-934.

3. Miller M, Stone NJ, Ballantyne C, et al. Triglycerides and cardiovascular disease: a scientific statement from the American Heart Association. Circulation. 2011;123:2292-333.

4. Berglund L, Brunzell JD, Goldberg AC, et al. Evaluation and treatment of hypertriglyceridemia: an Endocrine Society clinical practice guideline. J Clin Endocrinol Metab. 2012;97(9): 2969-89.

5. Tricor [package insert]. North Chicago: AbbVie Inc.; 2013.

6. Trilipix [package insert]. North Chicago: AbbVie Inc.; 2015.

7. Agouridis AP, Tsimihodimos V, Filippatos TD, et al. The effects of rosuvastatin alone or in combination with fenofibrate or omega 3 fatty acids on inflammation and oxidative stress in patients with mixed dyslipidemia. Expert Opin Pharmacother. 2011;12(17): 2605-11.

8. Ouwens MJ, Nauta J, Ansquer JC, et al. Systematic literature review and meta-analysis of dual therapy with fenofibrate or fenofibric acid and a statin versus a double or equivalent dose of statin monotheraphy. Curr Med Res Opin. 2015;31(12):2273-85.

9. Vascepa [package insert]. Bedminster: Amarin Pharma Inc.; 2015.

10. Bays HE, Ballantyne CM, Kastelein JJ, et al. Eicosapentaenoic acid ethyl ester (AMR101) therapy in patients with very high triglyceride levels (from the Multi-center, plAcebo-controlled, Randomized, double-blINd, 12-week study with an open-label Extension [MARINE] trial). Am J Cardiol. 2011;108(5): 682-90.

11. Ballantyne CM, Bays HE, Kastelein JJ, et al. Efficacy and safety of eicosapentaenoic acid ethyl ester (AMR101) therapy in statintreated patients with persistent high triglycerides (from the ANCHOR study). Am J Cardiol. 2012;110(7):984-92. 
12. Vascular disease in Western New York. Jacobs Neurological Institute, 2014. http://vps2836.inmotionhosting.com/ jacobsin/ images/PDF/Vascular\%20Disease\%20Infographics\%202014.pdf. Accessed 27 Jan 2016.

13. Friedewald WT, Levy RI, Fredrickson DS. Estimation of the concentration of low-density lipoprotein cholesterol in plasma, without use of the preparative ultracentrifuge. Clin Chem. 1972;18(6):499-502.

14. American Heart Association. Third report of the National Cholesterol Education Program (NCEP) expert panel on detection, evaluation, and treatment of high blood cholesterol in adults (Adult Treatment Panel III) final report. Circulation. 2002;106(25):3143-421.

15. Lipitor [package insert]. New York: Parke-Davis; 2012.

16. Lescol XL [package insert]. East Hanover: Novartis Pharmaceuticals Corporation; 2012.

17. Mevacor [package insert]. Whitehouse Station: Merck \& Co., Inc.; 2012.

18. Ginsberg HN, Elam MB, Lovato LC, et al. Effects of combination lipid therapy in type 2 diabetes mellitus. $\mathrm{N}$ Engl $\mathrm{J}$ Med. 2010;362(17):1563-74.

19. Keech A, Simes RJ, Barter P, et al. Effects of long-term fenofibrate therapy on cardiovascular events in 9795 people with type 2 diabetes mellitus (the FIELD study): randomised controlled trial. Lancet. 2005;366(9500):1849-61.

20. Crestor [package insert]. Wilmington: AstraZeneca Pharmaceuticals; 2012.

21. Davidson MH, Rosenson RS, Maki KC, et al. Effects of fenofibric acid on carotid intima-media thickness in patients with mixed dyslipidemia on atorvastatin therapy: randomized, placebo-controlled study (FIRST). Arterioscler Thromb Vasc Biol. 2014;34(6):1298-306.

22. van Dam M, Stalenhoef AFH, Wittekoek J, et al. Efficacy of concentrated n-3 fatty acids in hypertriglyceridaemia: a comparison with gemfibrozil. Clin Drug Investig. 2001;21:175-81.

23. Stalenhoef AF, de Graaf J, Wittekoek ME, et al. The effect of concentrated n-3 fatty acids versus gemfibrozil on plasma lipoproteins, low density lipoprotein heterogeneity and oxidizability in patients with hypertriglyceridemia. Atherosclerosis. 2000;153(1):129-38.

24. Goldberg AC, Bays HE, Ballantyne CM, et al. Efficacy and safety of ABT-335 (fenofibric acid) in combination with atorvastatin in patients with mixed dyslipidemia. Am $\mathrm{J}$ Cardiol. 2009;103(4):515-22.

25. Jones PH, Davidson MH, Kashyap ML, et al. Efficacy and safety of ABT-335 (fenofibric acid) in combination with rosuvastatin in patients with mixed dyslipidemia: a phase 3 study. Atherosclerosis. 2009;204:208-15.

26. Mohiuddin SM, Pepine CJ, Kelly MT, et al. Efficacy and safety of ABT-335 (fenofibric acid) in combination with simvastatin in patients with mixed dyslipidemia: a phase 3 , randomized, controlled study. Am Heart J. 2009;157(1):195-203.

27. Ballantyne CM, Jones PH, Kelly MT, et al. Long-term efficacy of adding fenofibric acid to moderate-dose statin therapy in patients with persistent elevated triglycerides. Cardiovasc Drugs Ther. 2011;25(1):59-67.

28. Khera AV, Qamar A, Reilly MP, et al. Effects of niacin, statin, and fenofibrate on circulating proprotein convertase subtilisin/ kexin type 9 levels in patients with dyslipidemia. Am J Cardiol. 2015;115(2):178-82.

29. Troutt JS, Alborn WE, Cao G, et al. Fenofibrate treatment increases human serum proprotein convertase subtilisin kexin type 9 levels. J Lipid Res. 2010;51(2):345-51.

30. Konrad RJ, Troutt JS, Cao G. Effects of currently prescribed LDL-C-lowering drugs on PCSK9 and implications for the next generation of LDL-C-lowering agents. Lipids Health Dis. 2011;10:38.

31. Niaspan [package insert]. North Chicago: AbbVie Inc.; 2015.

32. Keene D, Price C, Shun-Shin MJ, et al. Effect on cardiovascular risk of high density lipoprotein targeted drug treatments niacin, fibrates, and CETP inhibitors: meta-analysis of randomised controlled trials including 117,411 patients. BMJ. 2014;349:g4379.

33. Suh HS, Hay JW, Johnson KA, et al. Comparative effectiveness of statin plus fibrate combination therapy and statin monotherapy in patients with type 2 diabetes: use of propensity-score and instrumental variable methods to adjust for treatment-selection bias. Pharmacoepidemiol Drug Saf. 2012;21(5):470-84.

34. U.S. Food and Drug Administration. Trilipix supplement approval letter. http://www.accessdata.fda.gov/scripts/cder/ drugsatfda/index.cfm?fuseaction=Search.DrugDetails. Accessed 22 Dec 2015.

35. Lovaza [package insert]. Research Triangle Park: GlaxoSmithKline; 2014.

36. Omtryg [package insert]. Arlington: Trygg Pharma, Inc.; 2014.

37. Epanova [package insert]. Wilmington: AstraZeneca Pharmaceuticals LP; 2014.

38. Jacobson TA, Glickstein SB, Rowe JD, et al. Effects of eicosapentaenoic acid and docosahexaenoic acid on low-density lipoprotein cholesterol and other lipids: a review. J Clin Lipidol. 2012;6(1):5-18.

39. Wei MY, Jacobson TA. Effects of eicosapentaenoic acid versus docosahexaenoic acid on serum lipids: a systematic review and meta-analysis. Curr Atheroscler Rep. 2011;13(6):474-83.

40. Hilleman DE, Malesker MA. Potential benefits of icosapent ethyl on the lipid profile: case studies. Clin Med Insights Cardiol. 2014;8:13-5.

41. Castaldo RS. A retrospective case series of the lipid effects of switching from omega-3 fatty acid ethyl esters to icosapent ethyl in hyperlipidemic patients. Postgrad Med. 2014;126(3):268-73.

42. Hassan A, Tajuddin N, Shaikh A. Retrospective case series of patients with diabetes or prediabetes who were switched from omega-3-acid ethyl esters to icosapent ethyl. Cardiol Ther. 2015;4(1):83-93.

43. Kedia AW, Lynch E. Effects of switching from omega-3-acid ethyl esters to icosapent ethyl in a statin-treated patient with elevated triglycerides. Postgrad Med. 2015;127(8):869-73.

44. Crandell JR. Reductions in low-density lipoprotein cholesterol and particle concentration and improvements in other parameters of dyslipidemia in a statin-treated patient with persistent dyslipidemia and high cardiovascular risk following switch from EPA + DHA (omega-3-acid ethyl esters) to high-purity EPA (icosapent ethyl) [poster]. Presented at: Annual Meeting of the American Academy of Family Physicians. Denver, CO; 2015.

45. Crandell J, Tartaglia C, Tartaglia J. Retrospective case series of lipid effects in patients switched from EPA + DHA (omega- $3^{-}$ acid ethyl esters) to high-purity EPA (icosapent ethyl) [poster no. 124]. Presented at: Annual Cardiovascular Disease Prevention Conference of the American Society for Preventive Cardiology, Boca Raton, FL; 2015.

46. Mozaffarian D, Wu JH. Omega-3 fatty acids and cardiovascular disease: effects on risk factors, molecular pathways, and clinical events. J Am Coll Cardiol. 2011;58(20):2047-67.

47. Mason RP, Jacob RF, Corbalan JJ, et al. Combination eicosapentaenoic acid and statin treatment reversed endothelial dysfunction in HUVECs exposed to oxidized LDL [abstract no. 160]. J Clin Lipidol. 2014;8:342-3.

48. Mason RP, Jacob R, Beauregard G, et al. Comparative lipid antioxidant effects of omega-3 fatty acids in combination with HMG-CoA reductase inhibitors [abstract]. J Clin Lipidol. 2011;5:201. 
49. Toyama K, Nishioka T, Isshiki A, et al. Eicosapentaenoic acid combined with optimal statin therapy improves endothelial dysfunction in patients with coronary artery disease. Cardiovasc Drugs Ther. 2014;28(1):53-9.

50. Sasaki J, Miwa T, Odawara M. Administration of highly purified eicosapentaenoic acid to statin-treated diabetic patients further improves vascular function. Endocr J. 2012;59(4):297-304.

51. Thies F, Garry JM, Yaqoob P, et al. Association of n-3 polyunsaturated fatty acids with stability of atherosclerotic plaques: a randomised controlled trial. Lancet. 2003;361(9356):477-85.

52. Nishio R, Shinke T, Otake H, et al. Stabilizing effect of combined eicosapentaenoic acid and statin therapy on coronary thin-cap fibroatheroma. Atherosclerosis. 2014;234(1):114-9.

53. Cawood AL, Ding R, Napper FL, et al. Eicosapentaenoic acid (EPA) from highly concentrated n-3 fatty acid ethyl esters is incorporated into advanced atherosclerotic plaques and higher plaque EPA is associated with decreased plaque inflammation and increased stability. Atherosclerosis. 2010;212(1):252-9.

54. Calder PC, Yaqoob P. Marine omega-3 fatty acids and coronary heart disease. Curr Opin Cardiol. 2012;27(4):412-9.

55. Bays HE, Ballantyne CM, Braeckman RA, et al. Icosapent ethyl, a pure ethyl ester of eicosapentaenoic acid: effects on circulating markers of inflammation from the MARINE and ANCHOR studies. Am J Cardiovasc Drugs. 2013;13(1):37-46.

56. Yamano T, Kubo T, Shiono Y, et al. Impact of eicosapentaenoic acid treatment on the fibrous cap thickness in patients with coronary atherosclerotic plaque: an optical coherence tomography study. J Atheroscler Thromb. 2015;22(1):52-61.

57. Uehara H, Miyagi N, Shimajiri M, et al. The additional effect of eicosapentanoic acid on coronary plaque stability in stable angina patients with statin use by optical coherence tomography analysis [abstract no. P5495]. Eur Heart J. 2013;34(Suppl 1):1011.

58. Niki T, Wakatsuki T, Yamaquchi K, et al. Effects of the addition of eicosapentaenoic acid to strong statin therapy on inflammatory cytokines and coronary plaque components assessed by integrated backscatter intravascular ultrasound. Circ J. 2016;80(2):450-60.

59. Domei T, Amemiya K, Enomoto S, et al. Eicosapentaenoic acid reduced the progression of the coronary atherosclerosis in the patients with optimal LDL cholesterol lowering therapy [abstract no. P689]. Eur Heart J. 2013;34(Suppl 1):137.

60. Gajos G, Rostoff P, Undas A, et al. Effects of polyunsaturated omega-3 fatty acids on responsiveness to dual antiplatelet therapy in patients undergoing percutaneous coronary intervention: the OMEGA-PCI (OMEGA-3 fatty acids after pci to modify responsiveness to dual antiplatelet therapy) study. J Am Coll Cardiol. 2010;55(16):1671-8.

61. Nomura S, Shouzu A, Omoto S, et al. Effects of eicosapentaenoic acid on endothelial cell-derived microparticles, angiopoietins and adiponectin in patients with type 2 diabetes. J Atheroscler Thromb. 2009;16(2):83-90.

62. U.S. Food and Drug Administration. Orange book: approved drug products with therapeutic equivalence evaluations. http://www. accessdata.fda.gov/scripts/cder/ob/. Accessed 22 Dec 2015.

63. U.S. Food and Drug Administration. Regulatory information: dietary supplement health and education act of 1994. http:// health.gov/dietsupp/ch1.htm. Accessed 22 Dec 2015.

64. Lopez JAG, Ito MK. PLA chapter update: prescription fish oil and Blue Cross of Idaho. Lipid Spin. 2010;8:32-4.

65. American Cancer Society. Dietary supplements: what is safe? http://m.cancer.org/acs/groups/cid/documents/webcontent/002385pdf.pdf. Accessed 22 Dec 2015.

66. Kleiner AC, Cladis DP, Santerre CR. A comparison of actual versus stated label amounts of EPA and DHA in commercial omega-3 dietary supplements in the United States. J Sci Food Agric. 2015;95(6):1260-7.

67. Albert BB, Derraik JG, Cameron-Smith D, et al. Fish oil supplements in New Zealand are highly oxidised and do not meet label content of n-3 PUFA. Sci Rep. 2015;5:7928.

68. Ritter JC, Budge SM, Jovica F. Quality analysis of commercial fish oil preparations. J Sci Food Agric. 2013;93(8):1935-9.

69. Shim SM, Santerre CR, Burgess JR, et al. Omega-3 fatty acids and total polychlorinated biphenyls in 26 dietary supplements. J Food Sci. 2003;68:2436-40.

70. Zargar A, Ito MK. Long chain omega-3 dietary supplements: a review of the National Library of Medicine Herbal Supplement Database. Metab Syndr Relat Disord. 2011;9(4):255-71.

71. Bradberry JC, Hilleman DE. Overview of omega-3 fatty acid therapies. P T. 2013;38(11):681-91.

72. Weitz D, Weintraub H, Fisher E, et al. Fish oil for the treatment of cardiovascular disease. Cardiol Rev. 2010;18(5):258-63.

73. Truong P, Johnson C, Gabriel D. Variability of cholesterol and saturated fat content in dietary supplements [abstract no. P72]. Circulation. 2007;115:e238.

74. Halvorsen BL, Blomhoff R. Determination of lipid oxidation products in vegetable oils and marine omega-3 supplements. Food Nutr Res. 2011;55. doi:10.3402/fnr.v55i0.5792

75. Yokoyama M, Origasa H, Matsuzaki M, et al. Effects of eicosapentaenoic acid on major coronary events in hypercholesterolaemic patients (JELIS): a randomised open-label, blinded endpoint analysis. Lancet. 2007;369(9567):1090-8.

76. Weintraub HS. Overview of prescription omega-3 fatty acid products for hypertriglyceridemia. Postgrad Med. 2014;126(7): 7-18.

77. Amarin Pharma Inc. A study of AMR101 to evaluate its ability to reduce cardiovascular events in high risk patients with hypertriglyceridemia and on statin (REDUCE-IT). http://clinicaltrials. gov/show/NCT01492361. Accessed 22 Dec 2015. 